\title{
Solvent-free synthesis and oxidative aromatization of diethyl-2,6-dimethyl-4-(1- phenyl-3-aryl-1H-pyrazol-4-yl)-1,4-dihydropyridine-3,5-dicarboxylates using hypervalent iodine (III) reagents
}

\author{
Parvin Kumar $^{\mathrm{a}^{*}}$, Khalid Hussain ${ }^{\mathrm{b}}$ and Ashwani Kumar
}

${ }^{a}$ Department of Chemistry, Kurukshetra University, Kurukshetra, Haryana -136119, India

${ }^{b}$ Mewat Engineering College (Wakf), Palla, Tehsil: Nuh, District Mewat, Haryana 122107, India

${ }^{c}$ Drug Discovery and Research Laboratory, Department of Pharmaceutical Sciences, Guru Jambheshwar University of Science \& Technology, Hissar, Haryana -125001, India

\section{H R O N I C L E}

Article history:

Received June 28, 2013

Received in Revised form

December 10, 2013

Accepted 30 January 2014

Available online

30 January 2014

Keywords:

Pyrazole

Hantzch-1,4-dihdropyridine

Hypervalent iodine (III) reagent

Oxidative aromatization

Solvent-free \begin{abstract}
A B S T R A C T
In this article, an efficient, environmentally benign, solvent-free synthesis of diethyl-2,6dimethyl-4-(1-phenyl-3-aryl-1H-pyrazol-4-yl)-1,4-dihydropyridine-3,5-dicarboxylates and their simple oxidative aromatization in presence of selected hypervalent iodine (III) reagents under solvent-free condition at room temperature is demonstrated. All reactions were carried out by grinding the reactant pyrazole substituted Hantzch-1,4-dihydropyridines and hypervalent iodine (III) reagent in a mortar with pestle. [Hydroxy(tosyloxy)iodo]benzene act as an more efficient oxidizing reagent in comparison to phenyliodine bistrifluoroacetate and iodobenzene diacetate in terms of reaction time and yields. The advantages of present protocol are the environment friendly, short reaction time, mild reaction conditions, and high yields of the products.
\end{abstract}

(C) 2014 Growing Science Ltd. All rights reserved.

\section{Introduction}

The exploration of privileged structures in drug discovery is rapidly emerging theme in medicinal chemistry ${ }^{1}$. Pyrazoles and their derivatives are important class of compounds in organic and medicinal chemistry due to their biological properties ${ }^{2}$ including anti-inflammatory, antimicrobial, analgesic, hypoglycaemic and non-nucleoside HIV-1 reverse transcriptase inhibitor properties. Pyridine and its derivatives are an important part of organic compounds that have significant place in medicinal chemistry ${ }^{3}$. Thus, the synthesis of highly substituted pyridines has attracted much attention, and a number of procedures have been developed ${ }^{4}$. Out of these trials, we selected the oxidative aromatization of 1,4-dihydropyridines (1,4-DHP's). The 1,4-DHP's and their oxidized derivatives belong to such immensely important class of heterocyclic systems, owing to their potent

* Corresponding author. Tel.: +91-9416955143

E-mail addresses: parvinchem@kuk.ac.in, parvinjangra@gmail.com (P. Kumar) 
antihypertensive activity ${ }^{5}$ and other biological utilities ${ }^{6}$. These compounds generally undergo oxidative metabolism in the liver by the action of cytochrome $\mathrm{p}-450$ to form the corresponding pyridine derivatives ${ }^{7}$. Due to the relevance of this oxidative event to the biological NADH redox process $^{4-7}$, this transformation has attracted the attention of several research groups ${ }^{7-42}$.

Out of these numerous oxidative protocols, we selected the aromatization of pyrazole substituted1,4-DHP with hypervalent iodine (III) reagents under solvent-free condition. Recently hypervalent iodine (III) reagents have gained much importance as an oxidizing reagent due to their environmentally benign properties and replacing the use of toxic transition metals involved in such processes ${ }^{43-46}$. Hypervalent iodine (III) reagents are sparingly soluble in common organic solvents and therefore solvent free reactions are developed ${ }^{47}$. Solvent free reactions are of great importance in order to minimize pollution and toxic waste ${ }^{48-50}$. Literature survey shows that many exothermic reactions can be accomplished in high yields by just grinding solids together using mortar and pestle, a technique known as 'Grindstone Chemistry' ${ }^{51}$. Reactions are initiated by grinding, with the transfer of very small amount of energy through friction. It is not only advantageous from the environmental point of view but also offers rate enhancement, less waste products and higher yields ${ }^{51}$.

Encouraged by these observation and in continuation of our earlier studies on the oxidative aromatization of 1,4-DHP,s ${ }^{39-42}$ and synthesis of biological active heterocyclic compounds ${ }^{52-53}$, we report herein, solvent free aromatization of diethyl 2,6-dimethyl-4-(1-phenyl-3-aryl-1H-pyrazol-4-yl)1,4-dihydropyridine-3,5-dicarboxylates (2a-g) to diethyl 2,6-dimethyl-4-(1-phenyl-3-aryl-1Hpyrazol-4-yl)pyridine-3,5-dicarboxylates (3a-g) in presence of hypervalent iodine (III) reagents [iodobenzene diacetate (IBD) or phenyliodine bistrifluoroacetate (PIFA) or [hydroxy(tosyloxy)iodo]benzene (HTIB)] using grindstone chemistry (Scheme 2).

\section{Results and Discussion}

\subsection{Synthesis of diethyl-2,6-dimethyl-4-(1-phenyl-3-aryl-1H-pyrazol-4-yl)-1,4-dihydropyridine-3,5- dicarboxylates (2a-g)}

Compounds 2a-g were synthesized by multi-components reactions of ammonium acetate, ethylacetoacetate, formyl pyrazole (1 a-g) and silica without solvent at $90{ }^{\circ} \mathrm{C}$ (Scheme 1). To optimize the reaction condition for the synthesis of 1,4-DHP,s (2a-g), 3-(4-nitrophenyl)-1-phenyl-1H-pyrazole4-carbaldehyde $\mathbf{1 b}(1 \mathrm{mmol})$, ethylacetoacetate $(2.1 \mathrm{mmol})$, ammonium acetate $(1.1 \mathrm{mmol})$, and silica (10 mole\%) were heated at $90{ }^{\circ} \mathrm{C}$ for $3 \mathrm{hrs}$ afforded the corresponding 1,4-DHP $\mathbf{2 b}$ in $85 \%$ yield. Then reaction was performed in ethanol at refluxed condition (Table 1) and it furnished $\mathbf{2 b}$ in $75 \%$ yield. Without silica, time of reaction increased and yield of product decreased. Thus the reaction conditions were optimized (Table 1). Using optimized reaction condition, pyrazole substituted 1,4DHP's were synthesized (80-90\%). No electronic effect of substituents of formyl pyrazole was observed. Results are summarized in Table 2.

\subsection{Oxidative aromatization of diethyl-2,6-dimethyl-4-(1-phenyl-3-aryl-1H-pyrazol-4-yl)-1,4- dihydropyridine-3,5-dicarboxylates (2a-g)}

The high oxidizing power of hypervalent iodine (III) reagents led us to hypothesis that these reagents can act as efficient oxidizing reagents for this protocol. Initially diethyl 2,6-dimethyl-4-(3(4-nitrophenyl)-1-phenyl-1H-pyrazol-4-yl)-1,4-dihydropyridine-3,5-dicarboxylate 2b has been used as a substrate to test the feasibility of hypervalent iodine (III) reagents as an oxidant (Scheme 2). To optimize the reaction condition, diethyl 2,6-dimethyl-4-(3-(4-nitrophenyl)-1-phenyl-1H-pyrazol-4yl)-1,4-dihydropyridine-3,5-dicarboxylate $2 \mathbf{b}(1.0 \mathrm{mmol})$ and HTIB $(1.1 \mathrm{mmol})$ were ground in a mortar by pestle. After $3 \mathrm{~min}$ the reaction mixture became wet and then we carried out grinding till completion of reaction (TLC). After usual workup, diethyl 2,6-dimethyl-4-(3-(4-nitrophenyl)-1- 
phenyl-1H-pyrazol-4-yl)pyridine-3,5-dicarboxylate (3a) was obtained in excellent yield (88\%). Then we performed the reaction of $\mathbf{2 b}$ with IBD and PIFA. The oxidative efficiency of IBD or PIFA at room temperature was investigated, and it was observed that oxidation of pyrazole substituted 1,4DHP,s did not proceed effectively at room temperature. A better activity of IBD and PIFA was obtained when the preheated 1,4-DHP $\mathbf{2 b}$ was ground with these reagents. To optimize the reaction condition for IBD, hantzch-1,4-DHP was taken in mortar and kept in an oven at $80{ }^{\circ} \mathrm{C}-90^{\circ} \mathrm{C}$ for 5 $\mathrm{min}$, then it was removed from the oven, added IBD $(1.1 \mathrm{mmol})$, and ground with pestle. After some time, exothermic reaction occurred with liberation of acetic acid and reaction mass was ground till the completion of reaction (TLC). Same procedure was adopted for the oxidative aromatization of $\mathbf{2 b}$ with PIFA apart from the temperature of the oven. For PIFA mediated oxidative aromatization, compound $\mathbf{2 b}$ was heated in oven at $50{ }^{\circ} \mathrm{C}-60{ }^{\circ} \mathrm{C}$ for $5 \mathrm{~min}$ and then it was ground with PIFA by pestle.
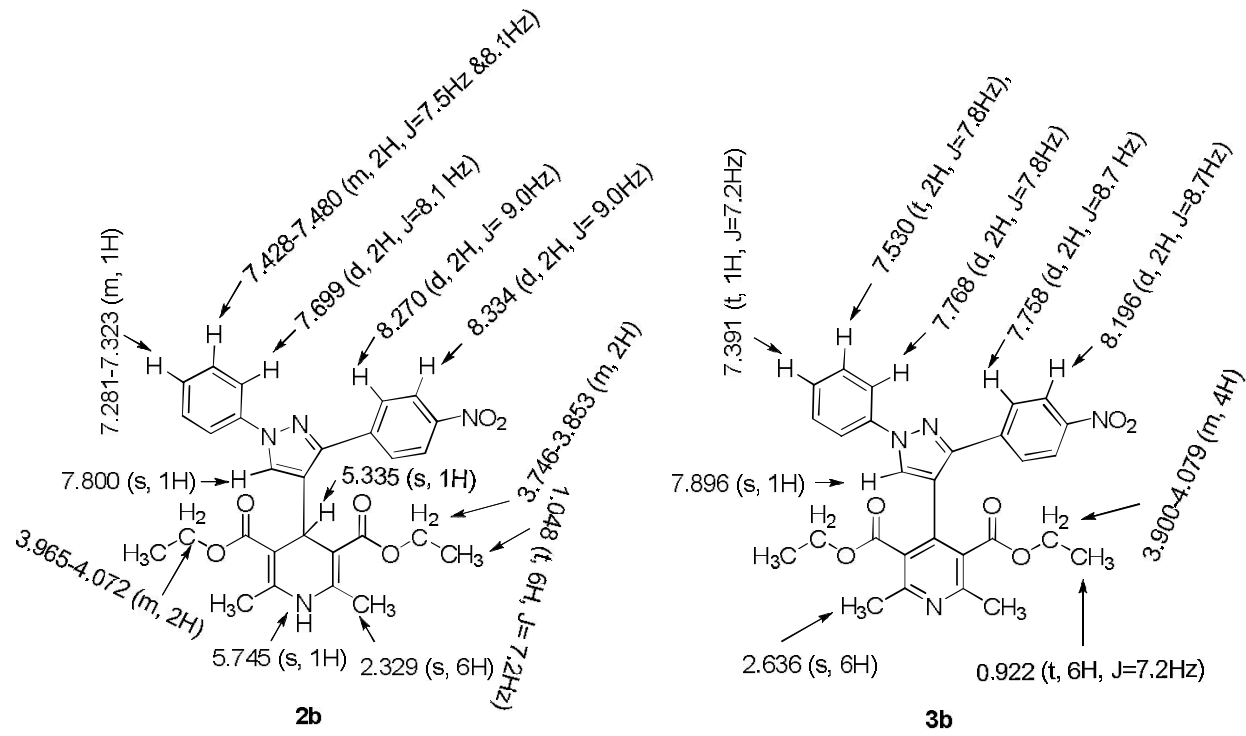

Fig.1. The hydrogen assignment in ${ }^{1} \mathrm{H}-\mathrm{NMR}$ spectra of $\mathbf{2} \mathbf{b}$ and $\mathbf{3 b}$

In ${ }^{1} \mathrm{H}-\mathrm{NMR}$ spectra of $\mathbf{2 b}$ the two separate multiplets appears at $\delta 3.746-3.853$ and $3.965-4.072$ for methylene protons but in ${ }^{1} \mathrm{H}-\mathrm{NMR}$ spectra of $\mathbf{3 b}$ these multiplets changes into a single multiplet at $\delta$ 3.900-4.079. Appearance of two multiplets in ${ }^{1} \mathrm{H}-\mathrm{NMR}$ spectra of $\mathbf{2 b}$ clearly indicates that both of the methylene groups are in different environment. This is further confirmed by finding most stable conformation of $\mathbf{2 b}$ with ChemBio 3D ultra 11.0 (Chem Bio Office 2008) and by using MM2 force field method of energy minimization (Fig. 2.).
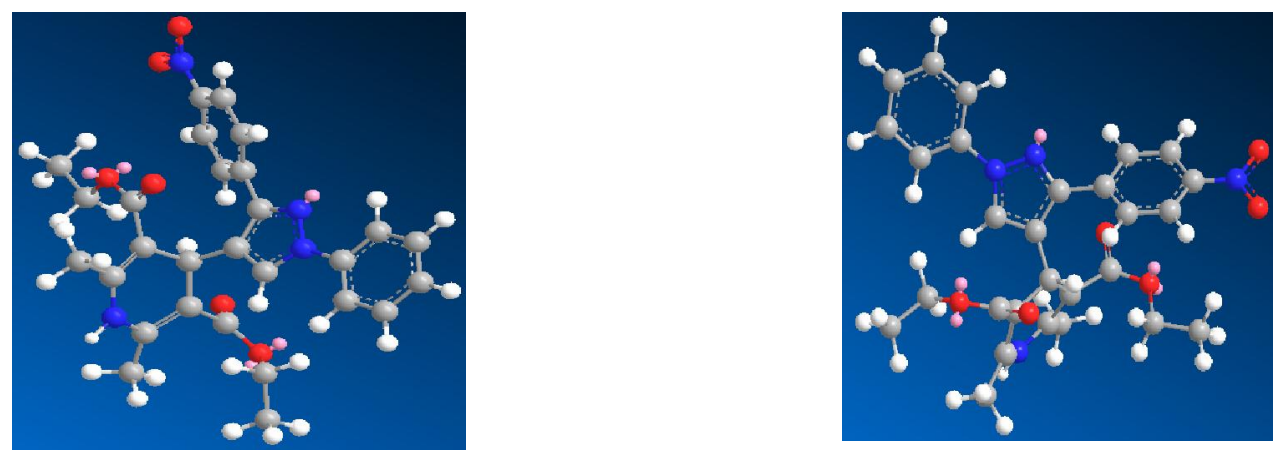

Fig. 2 Most stable conformation of $\mathbf{2 b}$

The total energy of the resulting conformation found for $\mathbf{2 b}$ is $24.2179 \mathrm{kcal} / \mathrm{mol}$. Appearance of one multiplet in ${ }^{1} \mathrm{H}-\mathrm{NMR}$ spectra of $\mathbf{3 b}$ shows that both of the methylene group are in similar 
environment (Fig. 3.). In ${ }^{1} \mathrm{H}-\mathrm{NMR}$ spectra of $\mathbf{3 b}$, the protons of methyl group, pyrazolyl proton, and aromatic proton of N-phenyl ring resonate at down field in comparison to $\mathbf{2} \mathbf{b}$ which evidently prove the electron withdrawing effect of $\mathrm{sp}^{2}$ hybridized nitrogen i.e. pyridine nitrogen. However, three types of proton (six protons of methyl group of ester, ortho and meta protons of $p$-nitrophenyl ring) resonate at high field.
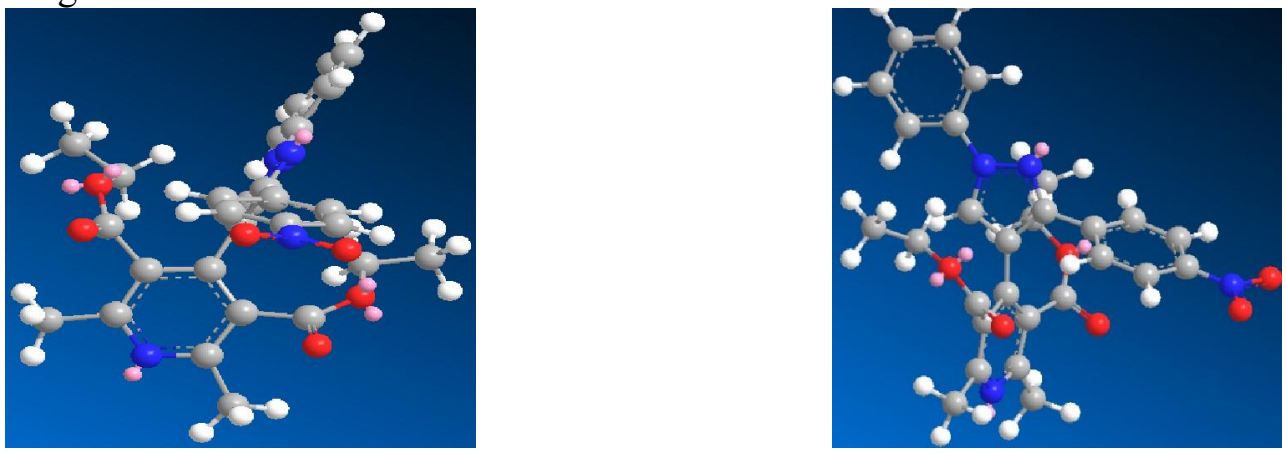

Fig. 3 Most stable conformation of $\mathbf{3 b}$

Under similar reaction conditions, various pyrazole substituted 1,4-dihydropyridines $\mathbf{2 a - g}$ were efficiently aromatized to the pyridine derivatives 3a-g and the results are given in Table 1. No electronic effect of 1,4-DHP's were observed.

Table 1. Silica-catalyzed synthesis of diethyl 4-(1,3-diphenyl-1 $H$-pyrazol-4-yl)-2,6-dimethyl-1,4dihydropyridine-3,5-dicarboxylate (2a) under different conditions.

\begin{tabular}{lllll}
\hline S. No. & Solvent & Amount of silica, mol $\%$ & Reaction temperature, ${ }^{\circ} \mathrm{C}$ & Yield, $\%$ \\
\hline 1 & None & None & 80 & 55 \\
2 & None & 5 & 80 & 65 \\
3 & None & 10 & 80 & 91 \\
4 & None & 15 & 80 & 90 \\
5 & None & 10 & 50 & 60 \\
6 & Ethanol & None & 80 & 50 \\
7 & Ethanol & 10 & 80 & 85 \\
\hline
\end{tabular}

Table 2. Silica-catalyzed synthesis of diethyl 4-(1-phenyl-3-aryl-1H-pyrazol-4-yl)-2,6-dimethyl-1,4dihydropyridine-3,5-dicarboxylates (2a-g).

\begin{tabular}{llllll}
\hline Entry & $\mathrm{R}$ & Time, & Yield, $\%$ & m.p., ${ }^{\circ} \mathrm{C}$ & Lit. m.p., ${ }^{\circ} \mathrm{C}$ \\
\hline $\mathbf{2 a}$ & $-\mathrm{H}$ & 3.0 & 90 & $167-169$ & $169-171^{54}$ \\
$\mathbf{2 b}$ & $-\mathrm{NO}_{2}$ & 3.5 & 85 & $123-125$ & $124-126^{54}$ \\
$\mathbf{2 c}$ & $-\mathrm{OCH}_{3}$ & 4.0 & 91 & $129-131$ & $128-130^{54}$ \\
$\mathbf{2 d}$ & $-\mathrm{CH}_{3}$ & 3.0 & 87 & $192-193$ & $194-195^{54}$ \\
$\mathbf{2 e}$ & $-\mathrm{Br}$ & 4.0 & 92 & $181-182$ & $174^{55}$ \\
$\mathbf{2 f}$ & $-\mathrm{Cl}$ & 4.0 & 90 & $166-168$ & $167-168^{54}$ \\
$\mathbf{2 g}$ & $-\mathrm{F}$ & 4.0 & 90 & $176-177$ & $175^{55}$ \\
\hline
\end{tabular}

Table 3. Solvent less oxidative aromatization of 4-(1-phenyl-3-aryl-1H-pyrazol-4-yl)-2,6-dimethyl1,4-dihydropyridine-3,5-dicarboxylates (2a-g).

\begin{tabular}{|c|c|c|c|c|c|c|c|c|c|}
\hline \multirow[t]{2}{*}{ Entry } & \multirow[t]{2}{*}{$\mathrm{R}$} & \multicolumn{2}{|c|}{ Reaction with IBD } & \multicolumn{2}{|c|}{ Reaction with PIFA } & \multicolumn{2}{|c|}{ Reaction with HTIB } & \multirow[t]{2}{*}{ m.p., ${ }^{\circ} \mathrm{C}$} & \multirow[t]{2}{*}{ Lit m.p., ${ }^{\circ} \mathrm{C}$} \\
\hline & & Time, ${ }^{a}$ min & Yield, \% & Time, ${ }^{\mathrm{b}} \min$ & Yield, \% & Time, ${ }^{c}$ min & Yield, \% & & \\
\hline $3 \mathbf{a}$ & $-\mathrm{H}$ & 20 & 70 & 15 & 77 & 10 & 90 & $114-116$ & $111^{55}$ \\
\hline 3b & $-\mathrm{NO}_{2}$ & 22 & 65 & 16 & 65 & 15 & 88 & $173-175$ & $172^{55}$ \\
\hline $3 c$ & $-\mathrm{OCH}_{3}$ & 23 & 67 & 18 & 69 & 15 & 90 & $138-139$ & $136^{55}$ \\
\hline 3d & $-\mathrm{CH}_{3}$ & 20 & 70 & 15 & 70 & 13 & 92 & $110-112$ & $105^{55}$ \\
\hline $3 \mathbf{e}$ & $-\mathrm{Br}$ & 25 & 72 & 18 & 70 & 14 & 87 & $116-118$ & $115^{55}$ \\
\hline $3 f$ & $-\mathrm{Cl}$ & 20 & 71 & 20 & 72 & 13 & 85 & $100-102$ & $101-102^{55}$ \\
\hline $3 g$ & $-F$ & 20 & 73 & 18 & 74 & 15 & 90 & $125-126$ & $121^{55}$ \\
\hline
\end{tabular}




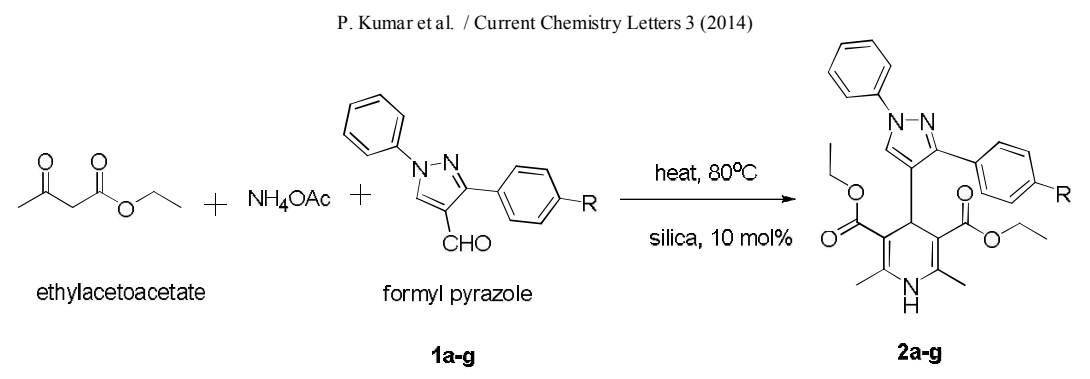

Scheme 1: Solvent-free synthesis of pyrazole substituted 1,4-DHP's

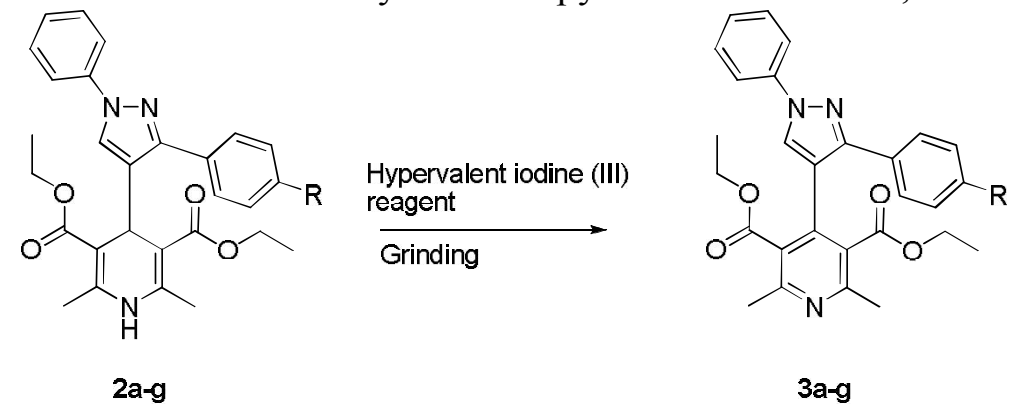

Scheme 2: Solvent-free oxidative aromatization of pyrazole substituted 1,4-DHP's.

\section{Conclusions}

In conclusion, in the present experiment, oxidation of 1,4-dihydropyridines with hypervalent iodine (III) reagents were performed efficiently. The hydroxy(tosyloxy)iodo]benzene (HTIB) was found more efficient reagent in comparison of IBD or PIFA. Literature survey ${ }^{30}$ and our present protocol also illustrate that efficiency of hypervalent iodine (III) reagents in studied oxidative aromatization of 1,4-DHP's is rose following HTIB > PIFA> IBD.

\section{Acknowledgements}

\section{Experimental}

\subsection{Materials and Methods}

All chemicals used in this study were of the highest purity available and purchased from local vendors. Melting points were determined on a Buchi oil heated melting apparatus and are uncorrected. ${ }^{1} \mathrm{H}-\mathrm{NMR}$ spectra were recorded in $\mathrm{CDCl}_{3}$ on a Bruker-300 $\mathrm{MHz}$ spectrometer using TMS as an internal standard (chemical shift in $\delta$ ). IR spectra were taken on a Perkin Elmer FTIR spectrophotometer using $\mathrm{KBr}$ pellets and peaks are reported in $\mathrm{cm}^{-1}$.

\subsubsection{General procedure for the synthesis of 1,4-dihydropyridines $\mathbf{2 a - g}$}

A mixture of pyrazole aldehyde $(5 \mathrm{mmol})$, ethyl acetoacetate $(10 \mathrm{mmol})$, ammonium acetate $(7.5$ $\mathrm{mmol})$ and silica $(10 \mathrm{~mol} \%)$ was heated at $90{ }^{\circ} \mathrm{C}$ for $3 \mathrm{hrs}$. Progress of reaction was monitored on TLC. After cooling, the reaction mixture was extracted with chloroform. Organic solvent was removed under vacuum. Then a yellowish colored solid mass was obtained and it was recrystallized with ethanol to get pure diethyl 1,4-dihydro-2,6-dimethyl-4-(3-aryl-1-phenyl-4-pyrazolyl)pyridine3,5-dicarboxylates (2a-g).

\subsubsection{General procedure for the oxidation of 1,4-dihydropyridines (2a-g) with HTIB}

A mixture of 1,4-dihydropyridine (2a-g) $(2 \mathrm{mmol})$ and HTIB (2.2 $\mathrm{mmol})$ was blended thoroughly in a mortar by pestle. The resulting homogeneous mixture was ground at room temperature for 5-10 
min. The completion of reaction was indicated by wetting of the reaction mixture. Progress of reaction was monitored by TLC. After completion of reaction, saturated solution of aq. sodium bicarbonate $(20 \mathrm{~mL})$ was added to quench the reaction and filtered the product. The resulting crude product was purified by silica gel column chromatography (using different ratios of ethyl acetate and $\mathrm{n}$-hexane as eluent according to different products).

\subsubsection{General procedure for the oxidation of 1,4-dihydropyridines (2a-g) with IBD or PIFA}

1,4-Dihdropydine was taken in mortar and heated in an oven by maintain the temperature of oven at $80-90^{\circ} \mathrm{C}$. After heating, mortar was taken off from the oven and IBD was added to it. Then the reaction mass was blended with pestle till the completion of reaction. Progress of reaction was monitored on TLC. If starting compound was observed on TLC then again the reaction mass was kept in oven for 2-3 min. After completion of reaction, saturated solution of aq. sodium bicarbonate (20 $\mathrm{mL}$ ) was added to quench the reaction and filtered the product. The resulting crude product was purified by silica gel column chromatography (using different ratios of ethyl acetate and n-hexane as eluent according to different products).

The same procedure was adopted for oxidative aromatization of 1,4-DHP with PIFA except the temperature of oven which was kept $50-60{ }^{\circ} \mathrm{C}$.

4.3.1. Spectral Data of diethyl-2,6-dimethyl-4-(3-aryl-1-phenyl-4-pyrazolyl)-1,4-dihydropyridine3,5-dicarboxylates (2a-g)

2a: IR (KBr): 3355, 3035, 2986, 1697, 1689, 1602, 1465, 1213, 750.

${ }^{1} \mathrm{H}-\mathrm{NMR}\left(\mathrm{CDCl}_{3}, \delta, \mathrm{ppm}\right): 1.092(\mathrm{t}, \mathrm{J}=6.9 \mathrm{~Hz}, 6 \mathrm{H}), 2.234(\mathrm{~s}, 6 \mathrm{H}), 3.744-3.848(\mathrm{~m}, 2 \mathrm{H}), 3.986-4.068$ $(\mathrm{m}, 2 \mathrm{H}), 5.307(\mathrm{~s}, 1 \mathrm{H}), 5.537(\mathrm{~s}, 1 \mathrm{H}), 7.221-7.279(\mathrm{~m}, 2 \mathrm{H}), 7.353-7.377(2 \mathrm{H}, \mathrm{d}, \mathrm{J}=7.2 \mathrm{~Hz}), 7.424-$ $7.447(2 \mathrm{H}, \mathrm{d}, \mathrm{J}=6.9 \mathrm{~Hz}) 7.760(\mathrm{~s}, 1 \mathrm{H}), 7.681-7.706(\mathrm{~d}, 2 \mathrm{H}, \mathrm{J}=7.5 \mathrm{~Hz}) ; 7.844-7.868(\mathrm{~d}, 2 \mathrm{H}, \mathrm{J}=7.2 \mathrm{~Hz})$.

Anal. Calcd for $\mathrm{C}_{28} \mathrm{H}_{29} \mathrm{~N}_{3} \mathrm{O}_{4}$. C, 71.32; H, 6.20; N, 8.91; Found: C, 71.45; H, 6.26; N 9.03.

2b: IR (KBr): 3385, 3077, 2991, 1696, 1681, 1623, 1543, 1467, 1338, 1235, 852, 720.

${ }^{1} \mathrm{H}-\mathrm{NMR}\left(\mathrm{CDCl}_{3}, \delta, \mathrm{ppm}\right): 1.048(\mathrm{t}, 6 \mathrm{H}, \mathrm{J}=7.2 \mathrm{~Hz}), 2.329(\mathrm{~s}, 6 \mathrm{H}), 3.746-3.853(\mathrm{~m}, 2 \mathrm{H}), 3.965-4.072$ $(\mathrm{m}, 2 \mathrm{H}), 5.335(\mathrm{~s}, 1 \mathrm{H}), 5.745(\mathrm{~s}, 1 \mathrm{H}), 7.281-7.323(\mathrm{~m}, 1 \mathrm{H}), 7.428-7.480(\mathrm{~m}, 2 \mathrm{H}, \mathrm{J}=7.5 \mathrm{~Hz} \& 8.1 \mathrm{~Hz})$, 7.699 (d, 2H, J=8.1 Hz), 7.800 (s, 1H), 8.270 (d, 2H, J=9.0Hz); 8.334 (d, 2H, J=9.0Hz).

Anal. Calcd for $\mathrm{C}_{28} \mathrm{H}_{28} \mathrm{~N}_{4} \mathrm{O}_{6}$. C, 65.11; H, 5.46; N, 10.85; Found: C, 65.29; H, 5.58; N 10.99.

2c: IR (KBr): 3322, 3033, 2981, 1696, 1683, 1621, 1451, 1220, 810.

${ }^{1} \mathrm{H}-\mathrm{NMR}\left(\mathrm{CDCl}_{3}, \delta, \mathrm{ppm}\right): 1.103(\mathrm{t}, 6 \mathrm{H}, \mathrm{J}=7.2 \mathrm{~Hz}), 2.784(\mathrm{~s}, 6 \mathrm{H}), 3.777-3.836(\mathrm{~m}, 2 \mathrm{H}), 3.866(\mathrm{~s}, 3 \mathrm{H})$, 3.995-4.100 (m, 2H), $5.288(\mathrm{~s}, 1 \mathrm{H}), 5.561(\mathrm{~s}, 1 \mathrm{H}), 6.977$ (d, 2H, J=8.7 Hz), $7.252(\mathrm{t}, 1 \mathrm{H}, \mathrm{J}=7.5 \mathrm{~Hz})$, 7.388-7.440 (m, 2H, J=7.5Hz \& J=7.8Hz ), 7.683 (d, 2H, J=7.8 Hz), $7.743(\mathrm{~s}, 1 \mathrm{H}), 7.800$ (d, 2H, J= $8.7 \mathrm{~Hz})$.

Anal. Calcd for $\mathrm{C}_{29} \mathrm{H}_{31} \mathrm{~N}_{3} \mathrm{O}_{5}$. C, 69.44; H, 6.23; N, 8.38; Found: C, 69.66; H, 6.39; N, 8.55.

2d: IR (KBr): 3347, 3066, 2992, 1695, 1685, 1610, 1597, 1442, 1211, 817, 720.

${ }^{1} \mathrm{H}-\mathrm{NMR}\left(\mathrm{CDCl}_{3}, \delta, \mathrm{ppm}\right): 1.087(\mathrm{t}, 6 \mathrm{H}, \mathrm{J}=7.2 \mathrm{~Hz}), 2.223(\mathrm{~s}, 6 \mathrm{H}), 2.403(\mathrm{~s}, 3 \mathrm{H}), 3.753-3.827(\mathrm{~m}, 2 \mathrm{H})$, 3.952-4.072 (m, 2H), $5.311(\mathrm{~s}, 1 \mathrm{H}), 5.724(\mathrm{~s}, 1 \mathrm{H}), 7.175-7.201(\mathrm{~d}, 2 \mathrm{H}, \mathrm{J}=7.8 \mathrm{~Hz}), 7.211(\mathrm{t}, 1 \mathrm{H}, \mathrm{J}=7.5$ $\mathrm{Hz}), 7.379-7.449$ (m, 2H), 7.678 (d, 2H, J=8.4 Hz), $7.761(\mathrm{~s}, 1 \mathrm{H}), 7.832-7.858$ (d, 2H, J= 7.8 Hz).

Anal. Calcd for $\mathrm{C}_{29} \mathrm{H}_{31} \mathrm{~N}_{3} \mathrm{O}_{4}$. C, 71.73; H, 6.43; N, 8.65; Found: C, 71.98; H, 6.55; N 8.72. 
2e: IR (KBr): 3399, 3063, 2981, 1695, 1683, 1612, 1466, 1208, 842.

${ }^{1} \mathrm{H}-\mathrm{NMR}\left(\mathrm{CDCl}_{3}, \delta, \mathrm{ppm}\right): 1.096(\mathrm{t}, 6 \mathrm{H}, \mathrm{J}=7.2 \mathrm{~Hz}), 2.277(\mathrm{~s}, 6 \mathrm{H}), 3.763-3.870(\mathrm{~m}, 2 \mathrm{H}), 3.998-$ $4.103(\mathrm{~m}, 2 \mathrm{H}), 5.281(\mathrm{~s}, 1 \mathrm{H}), 5.584(\mathrm{~s}, 1 \mathrm{H}), 7.257(\mathrm{t}, 1 \mathrm{H}, \mathrm{J}=7.2 \mathrm{~Hz}), 7.400-7.451(\mathrm{~m}, 2 \mathrm{H}, \mathrm{J}=7.5 \mathrm{~Hz}$ \& $7.8 \mathrm{~Hz}) ; 7.575(\mathrm{~d}, 2 \mathrm{H}, \mathrm{J}=8.1 \mathrm{~Hz}), 7.678(\mathrm{~d}, 2 \mathrm{H}, \mathrm{J}=7.8 \mathrm{~Hz}) 7.753(\mathrm{~s}, 1 \mathrm{H}), 7.820(\mathrm{~d}, 2 \mathrm{H}, \mathrm{J}=8.4 \mathrm{~Hz})$.

Anal. Calcd for $\mathrm{C}_{28} \mathrm{H}_{28} \mathrm{BrN}_{3} \mathrm{O}_{4}$. C, 61.10; H, 5.13; N, 7.63; Found: C, 61.22; H, 5.27; N, 7.72.

2f: IR (KBr): 3344, 3074, 2979, 1697, 1682, 1603, 1470, 1222, 837.

${ }^{1} \mathrm{H}-\mathrm{NMR}\left(\mathrm{CDCl}_{3}, \delta, \mathrm{ppm}\right): 1.096(\mathrm{t}, 6 \mathrm{H}, \mathrm{J}=7.2 \mathrm{~Hz}), 2.278(\mathrm{~s}, 6 \mathrm{H}), 3.766-3.837(\mathrm{~m}, 2 \mathrm{H}) ; 3.997-4.104$ $(\mathrm{m}, 2 \mathrm{H}), 5.285(\mathrm{~s}, 1 \mathrm{H}), 5.558(\mathrm{~s}, 1 \mathrm{H}), 7.235-7.260(\mathrm{~d}, 1 \mathrm{H}, \mathrm{J}=7.5 \mathrm{~Hz}), 7.406-7.454(\mathrm{~m}, 4 \mathrm{H}$,$) 7.668-7.75$ (m, 2H, J=7.8 Hz \& $6.9 \mathrm{~Hz}), 7.814(\mathrm{~s}, 1 \mathrm{H}), 7.863-7.891$ (d, 2H, J= 8.4 Hz).

Anal. Calcd for $\mathrm{C}_{28} \mathrm{H}_{28} \mathrm{~N}_{3} \mathrm{O}_{4}$ Cl. C, 66.47; H, 5.54; N, 8.31; Found: C, 66.47; H, 5.55; N, 8.31.

2g: IR (KBr): 3357, 3051, 2989, 1694, 1680, 1609, 1466, 1219, 831; ${ }^{1} \mathrm{H}-\mathrm{NMR}\left(\mathrm{CDCl}_{3}, \delta\right.$, ppm): $1.040(\mathrm{t}, 6 \mathrm{H}, \mathrm{J}=7.2 \mathrm{~Hz}), 2.519(\mathrm{~s}, 6 \mathrm{H}), 3.748-3.854(\mathrm{~m}, 2 \mathrm{H}), 3.964-4.070(\mathrm{~m}, 2 \mathrm{H}), 5.170(\mathrm{~s}, 1 \mathrm{H}), 5.559$ (s, 1H), 7.293 (d, 2H, J=7.5 Hz), 7.334-7.361 (m, 1H), 7.490-7.513 (m, 4H), 7.747 (d, 2H, J= 7.5 Hz), $7.920(\mathrm{~s}, 1 \mathrm{H})$.

Anal. Calcd for $\mathrm{C}_{28} \mathrm{H}_{28} \mathrm{~N}_{3} \mathrm{O}_{4}$ F. C, 68.70; H, 5.77; N, 8.58; Found: C, 68.88; H, 5.92; N 8.68. 4.3.2. Characterization data of dimethyl 2,6-dimethyl-4-pyrazolylpyridine-3,5-dicarboxylates (3a-g)

3a: IR (KBr): 3053, 3021, 2988, 1735, 1621, 1598, 1471, 1220, 745.

${ }^{1} \mathrm{H}-\mathrm{NMR}\left(\mathrm{CDCl}_{3}, \delta, \mathrm{ppm}\right): 0.943(\mathrm{t}, 6 \mathrm{H}, \mathrm{J}=7.2), 2.621(\mathrm{~s}, 6 \mathrm{H}), 3.912-4.081(\mathrm{~m}, 4 \mathrm{H}), 7.108-7.309$ (m, $4 \mathrm{H}), 7.816(\mathrm{~s}, 1 \mathrm{H}), 7.582-7.689(\mathrm{~m}, 6 \mathrm{H})$.

Anal. Calcd for $\mathrm{C}_{28} \mathrm{H}_{27} \mathrm{~N}_{3} \mathrm{O}_{4}$ : C, 71.62; H, 5.80; N, 8.95. Found: C, 71.84; H, 6.04; N 9.11.

3b: Yield- 68\%; IR (KBr): 1728, 1234, 1034.

${ }^{1} \mathrm{H}-\mathrm{NMR}\left(\mathrm{CDCl}_{3}, \delta, \mathrm{ppm}\right): 0.922(\mathrm{t}, 6 \mathrm{H}, \mathrm{J}=7.2 \mathrm{~Hz}), 2.636(\mathrm{~s}, 6 \mathrm{H}), 3.900-4.079(\mathrm{~m}, 4 \mathrm{H}), 7.391(\mathrm{t}, 1 \mathrm{H}$, $\mathrm{J}=7.2 \mathrm{~Hz}), 7.530(\mathrm{t}, 2 \mathrm{H}, \mathrm{J}=7.8 \mathrm{~Hz}), 7.758(\mathrm{~d}, 2 \mathrm{H}, \mathrm{J}=8.4 \mathrm{~Hz}), 7.768(\mathrm{~d}, 2 \mathrm{H}, \mathrm{J}=7.8 \mathrm{~Hz}), 7.896(\mathrm{~s}, 1 \mathrm{H})$, $8.196(\mathrm{~d}, 2 \mathrm{H}, \mathrm{J}=8.7 \mathrm{~Hz})$.

Anal. Calcd for $\mathrm{C}_{28} \mathrm{H}_{26} \mathrm{~N}_{4} \mathrm{O}_{6}: \mathrm{C}, 65.36 ; \mathrm{H}, 5.09 ; \mathrm{N}, 10.89$. Found: $\mathrm{C}, 65.55 ; \mathrm{H}, 5.21 ; \mathrm{N} 11.02$.

3c: IR (KBr): 3051, 2994, 1737, 1611, 1588, 1452, 832.

${ }^{1} \mathrm{H}-\mathrm{NMR}\left(\mathrm{CDCl}_{3}, \delta, \mathrm{ppm}\right): 0.948(\mathrm{t}, 6 \mathrm{H}, \mathrm{J}=7.2 \mathrm{~Hz}), 2.784(\mathrm{~s}, 6 \mathrm{H}), 3.832(\mathrm{~s}, 3 \mathrm{H}), 3.917-4.049(\mathrm{~m}, 4 \mathrm{H})$, $6.876(\mathrm{~d}, 2 \mathrm{H}, \mathrm{J}=9.0 \mathrm{~Hz}), 7.350(\mathrm{t}, 1 \mathrm{H}, \mathrm{J}=7.2 \mathrm{~Hz}), 7.425(\mathrm{~d}, 2 \mathrm{H}, \mathrm{J}=9.0 \mathrm{~Hz}) ; 7.475-7.528(\mathrm{~m}, 2 \mathrm{H}$, $\mathrm{J}=7.5 \mathrm{~Hz} \& 7.8 \mathrm{~Hz}) ; 7.755(\mathrm{dd}, 2 \mathrm{H}, \mathrm{J}=2.4 \mathrm{~Hz} \& \mathrm{~J}=7.5 \mathrm{~Hz}) 7.945(\mathrm{~s}, 1 \mathrm{H})$.

Anal. Calcd for $\mathrm{C}_{29} \mathrm{H}_{29} \mathrm{~N}_{3} \mathrm{O}_{5}: \mathrm{C}, 69.72 ; \mathrm{H}, 5.85 ; \mathrm{N}, 8.41$. Found: $\mathrm{C}, 69.86 ; \mathrm{H}, 5.99 ; \mathrm{N}$ 8.51.

3d: IR (KBr): 3067, 3008, 2991, 1741, 1617, 1590, 1445, 851, 719.

${ }^{1} \mathrm{H}-\mathrm{NMR}\left(\mathrm{CDCl}_{3}, \delta, \mathrm{ppm}\right): 0.937(\mathrm{t}, 6 \mathrm{H}, \mathrm{J}=7.2 \mathrm{~Hz}), 2.389(\mathrm{~s}, 3 \mathrm{H}), 2.531(\mathrm{~s}, 6 \mathrm{H}), 3.921-4.070(\mathrm{~m}, 4 \mathrm{H})$, $7.256(\mathrm{~d}, 2 \mathrm{H}, \mathrm{J}=7.8 \mathrm{~Hz}), 7.323-7.449(\mathrm{~m}, 3 \mathrm{H}), 7.715$ (d, 2H, J=8.2 Hz), $7.858(\mathrm{~d}, 2 \mathrm{H}, \mathrm{J}=7.8 \mathrm{~Hz})$, $7.931(\mathrm{~s}, 1 \mathrm{H})$.

Anal. Calcd for $\mathrm{C}_{29} \mathrm{H}_{29} \mathrm{~N}_{3} \mathrm{O}_{4}: \mathrm{C}, 72.03 ; \mathrm{H}, 6.04 ; \mathrm{N}, 8.69$. Found: $\mathrm{C}, 71.91 ; \mathrm{H}, 5.98 ; \mathrm{N} 8.81$.

3e: IR (KBr): 3036, 2982, 2899, 1745, 1601, 1559, 1502, 1441, 1379, 1339, 1296, 1238, 1205, 1091, $1039,1007,956,863,831,754,730,691 \mathrm{~cm}^{-1}$. 
${ }^{1} \mathrm{H}-\mathrm{NMR}\left(\mathrm{CDCl}_{3}, \delta, \mathrm{ppm}\right): 0.938(\mathrm{t}, 6 \mathrm{H}, \mathrm{J}=7.2 \mathrm{~Hz}), 2.615(\mathrm{~s}, 6 \mathrm{H}), 3.990-4.098(\mathrm{~m}, 4 \mathrm{H}), 7.310-7.372$ (m, 2H); 7.474-7.524 (m, 4H), 7.745 (d, 2H, J=7.8Hz), 7.922 (s, 1H).

Anal. Calcd for $\mathrm{C}_{28} \mathrm{H}_{26} \mathrm{~N}_{3} \mathrm{O}_{4} \mathrm{Br}$ : C, 61.42; H, 4.75; N, 7.68. Found: C, 61.31; H 4.79; N 7.69

3f: $\operatorname{IR}(\mathrm{KBr}): 3065,2994,2899,1742,1611,1595,1498,1464,1371,1319,1208,1088,1012,957$, $857,833,693 \mathrm{~cm}^{-1}$.

${ }^{1} \mathrm{H}-\mathrm{NMR}\left(\mathrm{CDCl}_{3}, \delta, \mathrm{ppm}\right): 0.940(\mathrm{t}, 6 \mathrm{H}, \mathrm{J}=7.2 \mathrm{~Hz}), 2.620(\mathrm{~s}, 6 \mathrm{H}), 3.898-4.118(\mathrm{~m}, 4 \mathrm{H}), 7.310-7.370$ $(\mathrm{m}, 2 \mathrm{H}) ; 7.487-7.513(\mathrm{~m}, 4 \mathrm{H}), 7.746(\mathrm{~d}, 2 \mathrm{H}, \mathrm{J}=7.8 \mathrm{~Hz}), 7.923(\mathrm{~s}, 1 \mathrm{H})$.

Anal. Calcd for $\mathrm{C}_{28} \mathrm{H}_{26} \mathrm{ClN}_{3} \mathrm{O}_{4}$ : C, 66.73; H, 5.20; N, 8.34. Found: C, 61.88; H, 5.31; N 8.49.

3g: IR (KBr): 3072, 3018, 2987, 1742, 1615, 1581, 1461, 1212, 1021, 838, 720.

${ }^{1} \mathrm{H}-\mathrm{NMR}\left(\mathrm{CDCl}_{3}, \delta, \mathrm{ppm}\right): 0.947$ (t, 6H, J=7.2Hz), $2.617(\mathrm{~s}, 6 \mathrm{H}), 3.899-4.110(\mathrm{~m}, 4 \mathrm{H}), 7.015(\mathrm{t}, 2 \mathrm{H}$, $\mathrm{J}=8.4 \mathrm{~Hz}), 7.341(\mathrm{t}, 1 \mathrm{H}, \mathrm{J}=7.2 \mathrm{~Hz}), 7.472(\mathrm{~d}, 2 \mathrm{H}, \mathrm{J}=7.8 \mathrm{~Hz}), 7.537(\mathrm{~d}, 2 \mathrm{H}, \mathrm{J}=8.4 \mathrm{~Hz}), 7.746(\mathrm{~d}, 2 \mathrm{H}$, $\mathrm{J}=7.8 \mathrm{~Hz}), 7.924(\mathrm{~s}, 1 \mathrm{H})$.

Anal. Calcd for $\mathrm{C}_{28} \mathrm{H}_{26} \mathrm{FN}_{3} \mathrm{O}_{4}$ : C, 68.98; H, 5.38; N, 8.62. Found: C, 69.08; H, 5.51; N 8.74.

\section{References}

1 Chikhale, R.V.; Bhole, R.P.; Khedekar, P.B.; Bhusari, K.P. (2009), Synthesis and pharmacological investigation of 3-(substituted 1-phenylethanone)-4-(substituted phenyl)-1, 2, 3, 4tetrahydropyrimidine-5-carboxylates. Eur. J. Med. Chem. 44, 3645.

2 Tewari, A. K.; Mishra, A. (2001), Synthesis and anti-inflammatory activities of $\mathrm{N}^{4}, \mathrm{~N}^{5}$-disubstituted3-methyl-1H-pyrazolo[3,4-c]pyridazines. Bioorg. Med. Chem. 9, 715.

3 Boger, D. L.; Nakahara, S. (1991), Diels-Alder reactions of N-sulfonyl-1-aza-1,3-butadienes: development of a synthetic approach to the streptonigrone C ring J. Org. Chem. 56, 880.

4 Fletcher, M. D.; Hurst, T. E.; Miles, T. J.; Moody, C. J. (2006), Synthesis of highly-functionalised pyridines via hetero-Diels-Alder methodology: reaction of 3-siloxy-1-aza-1,3-butadienes with electron deficient acetylenes. Tetrahedron 62, 5454.

5 Shan, R.; Howlett, S.E.; Knaus, E.E. (2002), Syntheses, Calcium Channel Agonist-Antagonist Modulation Activities, Nitric Oxide Release, and Voltage-Clamp Studies of 2-Nitrooxyethyl 1,4Dihydro- 2,6-dimethyl-3-nitro-4-(2-trifluoromethylphenyl)pyridine-5-carboxylate Enantiomers. $J$. Med. Chem. 45, 955 and references therein.

6 Safak, C.; Simsek, R. (2006), Fused 1,4-Dihydropyridines as Potential Calcium Modulatory Compounds. Mini-Rev. Med. Chem. 6, 747.

7 Adibia, H.; Hajipour, A. R. (2007), A convenient and efficient protocol for oxidative aromatization of Hantzsch 1,4-dihydropyridines using benzyltriphenylphosphonium peroxymonosulfate under almost neutral reaction conditions, Bio. Med. Chem. Lett. 17, 1008-1012.

8 Anniyappan, M.; Muralidharan, D.; Perumal, P. T. (2002), A novel application of the oxidizing properties of urea nitrate and peroxydisulfate-cobalt(II): Aromatization of NAD(P)H model Hantzsch 1,4-dihydropyridines, Tetrahedron 58, 5069-5073.

9 Chai, L.; Zhao, Y.; Sheng, Q.; Liu, Z. Q. (2006) Aromatization of Hantzsch 1,4-dihydropyridines and 1,3,5-trisubstituted pyrazolines with $\mathrm{HIO}_{3}$ and $\mathrm{I}_{2} \mathrm{O}_{5}$ in water, Tetrahedron Lett. 47, 9283-9285.

10 Chen, J. M.; Zeng, X.-M. (2009) $\beta$-Cyclodextrin-catalyzed mild aromatization of Hantzsch 1,4dihydropyridines with o-iodoxybenzoic acid in water/acetone. Synth. Commun. 39, 3521-3526.

11 Eynde, J. J. V.; Delfosse, F.; Mayence, A.; Van Haverbeke, Y. (1995) Old reagents, new results: Aromatization of Hantzsch 1,4-dihydropyridines with manganese dioxide and 2,3-dichloro-5,6dicyano-1,4-benzoquinone. Tetrahedron 51, 6511-6516.

12 Filipan-Litvic, M.; Litvic, M.; Vinkovic, V. (2008) An efficient, metal-free, room temperature aromatization of Hantzsch-1,4-dihydropyridines with urea-hydrogen peroxide adduct, catalyzed by molecular iodine. Tetrahedron 64, 5649-5656. 
13 Han, B.; Liu, Z.; Liu, Q.; Yang, L.; Liu, Z.; Yu, W. (2006) An efficient aerobic oxidative aromatization of Hantzsch 1,4-dihydropyridines and 1,3,5-trisubstituted pyrazolines. Tetrahedron 62, 2492-2496.

14 Hashemi, M. M.; Ahmadibeni, Y.; Ghafuri, H. (2003), Aromatization of Hantzsch 1,4dihydropyridines by hydrogen peroxide in the presence of cobalt(II) acetate. Monatsh Chem. 134, 107-110.

15 Heravi, M. M.; Behbahani, F. K.; Oskooie, H. A.; Shoar, R. H. (2005), Catalytic aromatization of Hantzsch 1,4-dihydropyridines by ferric perchlorate in acetic acid. Tetrahedron Lett. 46, 2775-2777.

16 Heravi, M. M.; Derikvand, F.; Hassan-Pour, S.; Bakhtiari, K.; Bamoharram, F. F.; Oskooie, H. A. (2007), Oxidative aromatization of Hantzsch 1,4-dihydropyridines in the presence of mixed-addenda vanadomolybdophosphate heteropolyacid $\mathrm{H}_{6} \mathrm{PMo}_{9} \mathrm{~V}_{3} \mathrm{O}_{40}$. Bio. Med. Chem. Lett. 17, 3305-3309.

17 Yadav, J. S.; Reddy, B. V. S.; Basak, A. K.; Baishya, G.; Narsaiah, A. V. (2006), Iodoxybenzoic Acid (IBX): An Efficient and Novel Oxidizing Agent for the Aromatization of 1,4-Dihydropyridines Synthesis 3, 451-454.

18 Khadilkar, B.; Borkar, S. (1998) Silica gel-supported ferric nitrate: A convenient oxidizing reagent, Synth. Commun. 28, 207-212.

19 Litvic, M.; Cepanec, I.; Filipan, M.; Kos, K.; Bartolincic, A.; Druskovic, V.; Tibi, M. M.; Vinkovic, V. (2005), Mild, selective, and high-yield oxidation of Hantzsch 1,4-dihydropyridines with lead(IV) acetate. Heterocycles 65, 23.

20 Liu, D.; Gui, J.; Wang, C.; Lu, F.; Yang, Y.; Sun, Z. (2010) Oxidative aromatization of Hantzsch 1,4dihydropyridines catalyzed by ferric perchlorate in ionic liquids with air. Synth. Commun. 40, 1004 1008.

21 Liu, Z.; Yu, W.; Yang, L.; Liu, Z.-L. (2007), A novel oxidation-ring contraction of Hantzsch 1,4dihydropyridines to polysubstituted furans, Tetrahedron Lett. 48, 5321.

22 Memarian, H. R.; Abdoli-Senejani, M. (2008), Ultrasound-assisted photochemical oxidation of unsymmetrically substituted 1,4-dihydropyridines. Ultrason. Sonochem. 15, 110.

23 Memarian, H. R.; Abdoli-Senejani, M.; Tangestaninejad, S. (2006), Photosensitized oxidation of unsymmetrical 1,4-dihydropyridines. J. Iran. Chem. Soc. 3, 285.

24 Moghadam, M.; Nasr-Esfahani, M.; Tangestaninejad, S.; Mirkhani, V. (2006), Mild and efficient oxidation of Hantzsch 1,4-dihydropyridines with sodium periodate catalyzed by a new polystyrenebound Mn(TPP)Cl. Bioorg. Med. Chem. Lett. 16, 2026.

25 Nasr-Esfahani, M.; Moghadam, M.; Tangestaninejad, S.; Mirkhani, V.; Momeni, A. R. (2006), Rapid and efficient oxidation of Hantzsch 1,4-dihydropyridines with sodium periodate catalyzed by manganese(III) Schiff base complexes. Bioorg. Med. Chem. 14, 2720.

26 Pfister, J. R. (1990), Rapid, high-yield oxidation of Hantzsch-type 1,4-dihydropyridines with ceric ammonium nitrate. Synthesis 689-690.

27 Saini, A.; Kumar, S.; Sandhu, J. S. (2007), New strategy for the oxidation of Hantzsch 1,4dihydropyridines and dihydropyrido[2,3- $d$ ] pyrimidines catalyzed by DMSO under aerobic conditions. Synth. Commun. 37, 2317-2324.

28 Sharma, S. D.; Hazarika, P.; Konwar, D. (2008), A simple, green, and one-pot, four-component synthesis of 1,4-dihydropyridines and their aromatization. Catal. Commun. 9, 709-714.

29 Tamaddon, F.; Razmi, Z. (2011), Oxidation of 1,4-dihydropyrimidines and 3,4-dihydropyrimidin$2(1 \mathrm{H})$-ones to substituted pyridines and pyrimidinones using $\mathrm{Ca}(\mathrm{OCl})_{2}$ in aqueous media. Synth. Commun. 41, 485-492.

30 Eynde, J. V.; Dorazio, R.; Haverbeke, Y. V. (1994), Potassium permanganate, a versatile reagent for the aromatization of Hantzsch 1,4-dihydropyridines. Tetrahedron 50, 2479-2484.

31 Varma, R. S.; Kumar, D. (1999), Manganese triacetate- mediated oxidation of Hantzsch 1,4dihydropyridines to pyridines. Tetrahedron Lett. 40, 21-24.

32 Xia, J. J.; Wang, G. W. (2005), One-pot synthesis and aromatization of 1,4-dihydropyridines in refluxing water, Synthesis 2379-2383.

33 Zeynizadeh, B.; Dilmaghani, K. A.; Mirzaei, M. (2007), Mild and convenient method for aromatization of Hantzsch esters of 1,4-dihydropyridines with $\mathrm{Ag}_{2} \mathrm{O}$. Acta. Chim. Slov. 54, 366.

34 Karade, N. N.; Gampawar, S. V.; Kondre, J. M.; Shinde, S. V. (2008), An efficient combination of Dess-Martin periodinane with molecular iodine or $\mathrm{KBr}$ for the facile oxidative aromatization of Hantzsch 1, 4-dihydropyridines. ARKIVOC xii, 9-16. 
35 Cheng, D. P.; Chen, Z. C. (2002), Hypervalent iodine in synthesis 76. An efficient oxidation of 1,4dihydropyridines to pyridines using iodobenzene diacetate. Synth. Commun. 32, 793-798.

36 Lee, K.H.; Ko, K.Y. (2002), Aromatization of Hantzsch 1,4-Dihydropyridines with [Hydroxy(tosyloxy)iodo]benzene. Bull. Korean Chem. Soc. 23, 1505-1506.

37 Lee, J. W.; Ko, K. Y. (2004), Aromatization of Hantzsch 1,4-Dihydropyridines with a Polymersupported Hypervalent Iodine Reagent. Bull. Korean Chem. Soc. 25, 19-20.

38 Varma, R. S.; Kumar, D. (1999), Solid state oxidation of 1,4-dihydropyridines to pyridines using phenyliodine(III) bis(trifluoroacetate) or elemental sulfur. J. Chem. Soc. Perkin Trans 1 1755-1757.

39 Kumar, P. (2009), Solid State Oxidative Aromatization of Hantzsch 1,4-Dihydropyridines to Pyridines Using Iodobenzene Diacetate or Hydroxy(tosyloxy)iodobenzene. Chin. J. Chem. 27, 1487-1491.

40 Kumar, P. (2010), A novel, facile, simple and convenient oxidative aromatization of Hantzsch 1,4dihydropyridines to pyridines using polymeric iodosobenzene with $\mathrm{KBr}$, J. Heterocycl. Chem. 47, 1429-1433.

41 Kumar, P.; Kumar, A. (2010) An Expeditious Oxidative Aromatization of Hantzsch 1,4Dihydropyridines to Pyridines Using Cetyltrimethylammonium Peroxodisulfate: A Phase Transferring Oxidant. Bull. Korean Chem. Soc. 31, 2299.

42 Kumar, P.; Kumar, A.; Hussain, K. (2012), Iodobenzene diacetate (IBD) catalyzed an quick oxidative aromatization of Hantzsch-1,4-dihydropyridines to pyridines under ultrasonic irradiation Ultrasonics Sonochem. 19, 729.

43 Varvoglis, A. (1997), Chemical transformations induced by hypervalent iodine reagents. Tetrahedron $53,1179-1255$.

44 Wirth, T.; Chiai, M.; Zhdankin, V.V.; Koser, G.F.; Tohma, H.; Kita, Y. (2003), Hypervalent iodine chemistry-modern developments in organic synthesis, Topics in Current Chemistry, Springer, Berlin vol. 224.

45 Zhdankin, V.V.; Stang, P.J. (2008), Chemistry of Polyvalent Iodine. Chem. Rev. 108, 5299.

46 Kumar, D.; Sundaree, M.S.; Patel, G.; Rao, V. S.; Varma, R. S. (2006), Solvent-free facile synthesis of novel $\alpha$-tosyloxy $\beta$-keto sulfones using [hydroxy(tosyloxy)iodo]benzene. Tetrahedron Lett. 47, 8239.

47 Yusubov, M. S.; Wirth, T. (2005), Solvent-Free Reactions with Hypervalent Iodine Reagents Org. Let. 7, 519 and references cited within.

48 Tanaka, K.; Toda, F. (2000), Solvent-Free Organic Synthesis. Chem. Rev. 100, 1025.

49 Cave, G. W. V.; Raston, C. L.; Scott, J. L. (2001) Recent advances in solventless organic reactions: towards benign synthesis with remarkable versatility Chem. Commun. 2159.

50 Varma, R. S. (1999), Solvent-free organic syntheses. using supported reagents and microwave irradiation, Green Chem. 1, 43.

51 Gupta, R.; Jain, A.; Jain, M.; Joshi, R. (2010) 'One Pot' Synthesis of 2-Amino-3-cyano-4,6diarylpyridines under Ultrasonic Irradiation and Grindstone Technology. Bull. Korean Chem. Soc. 31, 3180 .

52 Kumar, P.; Kumar, A.; Mohan, J.; Makrandi, J. K. (2010) Heterocyclic Systems Containing Bridgehead Nitrogen Atom: Synthesis and Evaluation of Biological Activity of Imidazo[2,1-b]-1,3,4thiadiazolo [2,3-c]-s-triazoles, s-Triazolo[3,4-b]-1,3,4-thiadiazolo[3,2-b]imidazo[4,5-b]quinoxaline and bis-(s-Triazolo[3,4-b]-1,3,4-thiadiazolo[3,2-b][imidazo[4,5-b]-cyclohexane]-5a,6a-diene). Bull. Korean Chem. Soc. 31, 3304.

53 Kumar, P.; Kumar, S.; Husain, K.; Kumar, A. (2011), An efficient synthesis of pyrazole chalcones under solvent-free conditions at room temperature. Chin. Chem. Lett. 22, 37.

54 Li, J.; Qiu, J.; Li, H.; Zhang, G. (2011), An Efficient, Three-component One-pot Preparation of 1,4Dihydropyridines Containing Novel Substituted Pyrazole under Sulfamic Acid Catalysis. Chin. J. Chem. 29, 511.

55 Prakash, O. ; Hussain, K. ; Kumar, R.; Wadhwa, D. ; Sharma, C. ; Aneja, K.R. (2011), Synthesis and antimicrobial evaluation of new 1,4-dihydro-4-pyrazolylpyridines and 4-pyrazolylpyridines. Org. Med. Chem. Lett. 1:5 doi:10.1186/2191-2858-1-5. 